\title{
Pilocarpine induces an increase in the anterior chamber angular width in eyes with narrow angles
}

\author{
Hiroshi Kobayashi, Kaori Kobayashi, Junichii Kiryu, Takehisa Kondo
}

\begin{abstract}
Aim-To determine the mechanical effects of pilocarpine on the trabecular-iris angle opening in eyes with narrow angles, compared with its effects on healthy control subjects with wide angles. Methods-A narrow angle was defined as $25^{\circ}$ or less of trabecular-iris angle on ultrasound biomicroscopic examination. The change in anterior chamber depth (ACD), trabecular-iris angle (TIA), angle opening distance (AOD, distance between trabecular meshwork and iris) measured at $250 \mu \mathrm{m}$ and $500 \mu \mathrm{m}$ from the scleral spur (AOD250 and AOD500), and iris thickness was determined in 30 eyes of 30 patients (13 men and 17 women, between 63 and 82 years (mean 70.4 years)) with narrow angles and in 30 sex and age matched control subjects with wide angles before and 1 hour after the instillation of $2 \%$ pilocarpine hydrochloride by ultrasound biomicroscopy.
\end{abstract}

Results-In all eyes with narrow angles, pilocarpine increased the TIA, AOD250, and AOD500; these changes increased significantly and linearly as the corresponding pretreatment values decreased $(r=$ $0.807, \mathrm{p}=0.0001 ; r=0.787, \mathrm{p}=0.0001 ; r=$ $0.852, p=0.0001)$. Of 30 eyes with wide angles, 23 eyes whose ACD was $2670 \mu \mathrm{m}$ and more showed a decrease in the TIA, AOD250, and AOD500; the changes in TIA, AOD250, and AOD500 also significantly correlated with the corresponding pretreatment values $(r=0.913, p=0.0001$; $r=0.882, \mathrm{p}=0.0001 ; r=0.895, \mathrm{p}=0.0001)$. Pilocarpine induced a smaller decrease in ACD in eyes with narrow angles than in those with wide angles $(p=0.0001)$. There was a linear correlation between the increase in ACD change and the decrease in pretreatment $A C D$ in eyes with narrow angles and those with wide angles $(r=$ $0.781, p=0.0003 ; r=0.798, p=0.0001$ ).

Conclusions-The finding that pilocarpine increases angular width in patients with narrow angles indicates that this agent is useful for treating patients with narrow angles and angle closure glaucoma. The prediction of the pilocarpine induced change in the angle may assist ophthalmologists in treating such patients.

(Br f Ophthalmol 1999;83:553-558)

Pilocarpine, one of the most commonly used and the most extensively studied antiglaucoma agents, reduces intraocular pressure by in- creasing aqueous outflow. ${ }^{1-6}$ The change in the anterior chamber angle results from dual responses to pilocarpine. One is a forward movement of the iris plane, which results from increased resistance to the flow from the posterior chamber to the anterior chamber, accommodation, and increased outflow. The other is contraction of the sphincter muscle of the iris, which leads to a decrease in total thickness and a pull on the root of the iris. The net response of the angle opening to pilocarpine is the combination of these opposite reactions: the narrowing by forward movement of the iris plane and widening by contraction of the sphincter muscle of the iris. ${ }^{7}$

We have previously reported that the response of the anterior chamber angle opening to pilocarpine varies according to the pretreatment status in healthy volunteers aged 50 and under; if the pretreatment angle was greater than $41.1^{\circ}$, pilocarpine decreased the angular width, and, if the pretreatment angle was less than $41.1^{\circ}$, pilocarpine increased the angular width. ${ }^{8}$ Understanding the response to pilocarpine in patients with narrow angles is essential for the treatment of angle closure and narrow angle glaucoma. The ability to predict the pilocarpine induced change in the angle before pilocarpine administration in such patients would be helpful for ophthalmologists.

Conventional methods such as gonioscopy and ultrasonic biometry present difficulties in evaluating fine structures of the anterior chamber, ${ }^{9-11}$ and their use can lead to an incorrect diagnosis. ${ }^{12}$ High resolution ultrasound biomicroscopy provides precise measurements of the subtle change of the angle induced by pilocarpine as well as images that cannot be obtained by conventional methods. ${ }^{13-21}$ Ritch followed patients who were judged at risk for developing angle closure glaucoma by their ophthalmologists and showed that this group had an almost $30 \%$ risk of developing angle closure within 5 years. ${ }^{7}$ Although prophylactic laser iridotomy treatment should be employed on the fellow eyes of patients who have suffered an attack of angle closure in one eye, its precise indications have not been established..$^{22}$

In the present study, we examined the angle change induced by pilocarpine in patients with narrow angles who underwent prophylactic laser iridotomy after this examination and compared the results of those of age matched control subjects before and after administration of pilocarpine using ultrasound biomicroscopy.

\section{Materials and methods}

We evaluated 30 eyes of 30 patients with narrow angles who underwent prophylactic
Accepted for publication 25 November 1998 

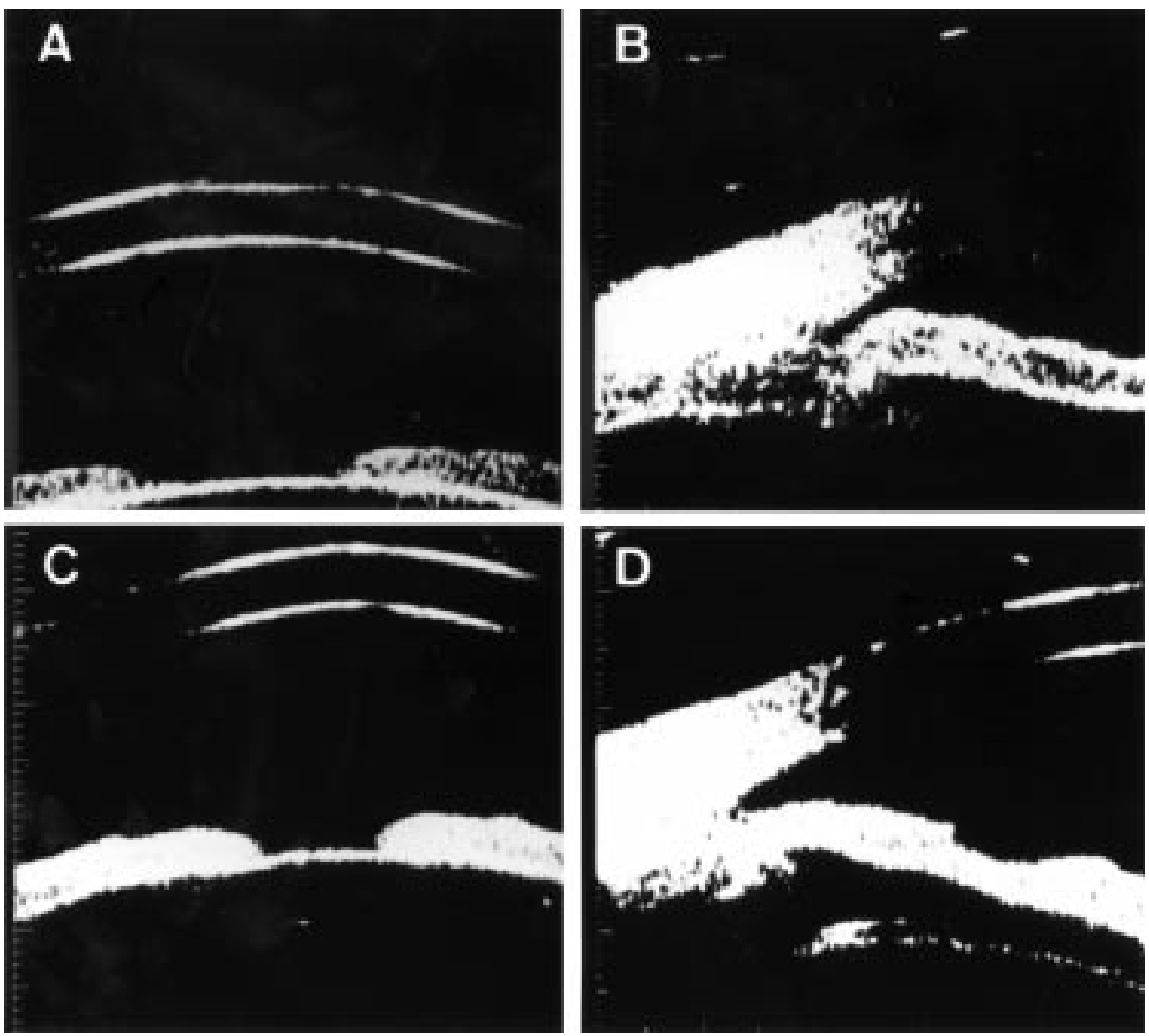

$E$

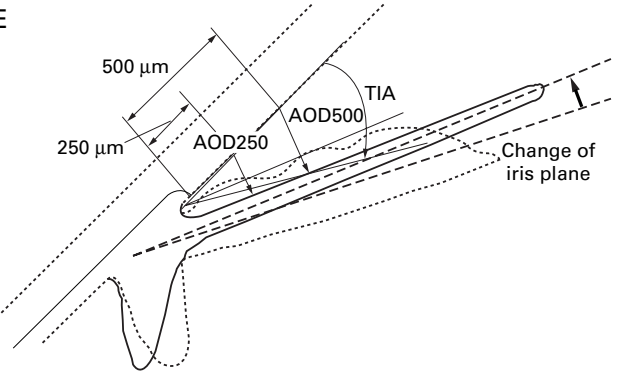

Figure 1 Change of narrow angle by pilocarpine. (A) (B) Before the instillation of pilocarpine. (C) (D) After the instillation of pilocarpine. (E) Graphic illustration of measurement of TIA, AOD250, and AOD500 on ultrasound biomicroscopic cross sectional views before and after pilocarpine. A broken line and a solid line indicate the state of the angle and the iris before and after pilocarpine. Lines with arrowheads show changes of TIA, AOD250, AOD500, and the iris plane.

Table 1 Comparison of change (SD) (range) in ACD, TIA, AOD250, AOD500, and iris thickness between wide angle and narrow angle groups

\begin{tabular}{|c|c|c|c|c|}
\hline Variable & Before pilocarpine & After pilocarpine & Change & p Value \\
\hline \multicolumn{5}{|l|}{ Wide angle group $(n=30)$ : } \\
\hline Age (years) & $70.42(2.91)(63$ to 85$)$ & - & - & \\
\hline Total axial length (mm) & $24.91(2.63)(21.46$ to 28.76$)$ & - & - & \\
\hline Lens thickness (mm) & $3.82(0.68)(2.48$ to 5.01$)$ & - & - & \\
\hline $\mathrm{ACD}(\mu \mathrm{m})$ & 2963 (267) (2506 to 3510$)$ & 2816 (262) (2392 to 3353$)$ & $-147(19)(-114$ to -190$)$ & \\
\hline TIA (degree) & $41.86(7.47)(25.72$ to 53.77$)$ & $38.42(3.77)(29.84$ to 45.54$)$ & $-3.44(4.82)(-12.31$ to +5.40$)$ & \\
\hline AOD $250(\mu \mathrm{m})$ & $338.1(70.6)(243$ to 508$)$ & $307.5(42.9)(240$ to 404$)$ & $-30.6(38.2)(-104$ to +53$)$ & \\
\hline AOD $500(\mu \mathrm{m})$ & $426.3(73.8)(285$ to 564$)$ & $392.0(42.9)$ (305 to 468$)$ & $-34.3(40.7)(-127$ to +32$)$ & \\
\hline Iris thickness $(\mu \mathrm{m})$ & 464.8 (69.6) (395 to 602$)$ & $358.3(68.0)$ (270 to 418$)$ & $-106.5(30.6)(-187$ to -50$)$ & \\
\hline $\operatorname{ILCD}(\mu \mathrm{m})$ & $1,223(657)(856$ to 1,765$)$ & 2,137 (433) $(1,876$ to 2,675$)$ & $914(543(532)$ to 1,451$)$ & \\
\hline ILCA $\left(\mathrm{mm}^{3}\right)$ & $20.512(7.833)(14.128$ to 29.325$)$ & 37.435 (3.357) (33.699 to 44.747$)$ & $16.923(5.795)(7.483$ to 23.619$)$ & \\
\hline \multicolumn{5}{|l|}{ Narrow angle group $(n=30)$ : } \\
\hline Age (years) & 71.56 (2.76) (65 to 85$)$ & - & - & 0.2498 \\
\hline Total axial length $(\mathrm{mm})$ & 22.46 (1.00) (21.33 to 24.22$)$ & - & - & 0.0017 \\
\hline Lens thickness (mm) & $4.88(0.40)(4.38$ to 5.46$)$ & - & - & 0.0001 \\
\hline $\mathrm{ACD}(\mu \mathrm{m})$ & 2055 (214) (1706 to 2470$)$ & 1961 (202) (1623 to 2360$)$ & $-94(15)(-67$ to -120$)$ & 0.0001 \\
\hline TIA (degree) & $16.79(7.751)(3.32$ to 30.22$)$ & $24.73(5.75)(14.79$ to 34.11$)$ & $+7.94(2.77)(+2.41$ to +13.21$)$ & 0.0001 \\
\hline AOD $250(\mu \mathrm{m})$ & $144.8(69.8)(32$ to 278$)$ & $216.5(48.9)(130$ to 315$)$ & $+71.7(29.1)(+13$ to +120$)$ & 0.0001 \\
\hline AOD500 $(\mu \mathrm{m})$ & $195.7(90.3)(49$ to 367$)$ & $278.7(61.8)(181$ to 382$)$ & $+83.0(37.2)(+7$ to +142$)$ & 0.0001 \\
\hline Iris thickness $(\mu \mathrm{m})$ & $459.2(49.8)(228$ to 449$)$ & $318.8(78.8)(228$ to 440$)$ & $-140.4(26.3)(46$ to 132$)$ & 0.1253 \\
\hline ILCD $(\mu \mathrm{m})$ & $1,856(534)(1,356$ to 2,165$)$ & $2,721(378)(2,034$ to 3,432$)$ & $865(467(676)$ to 1,243$)$ & 0.3747 \\
\hline ILCA $\left(\mathrm{mm}^{3}\right)$ & $35.002(7.203)(23.656$ to 44.103$)$ & $45.638(7.686)(30.874$ to 60.693$)$ & $10.626(7.585)(4.829$ to 18.367$)$ & 0.0006 \\
\hline
\end{tabular}

Values are means (SD) examined by Student's $t$ test and Welch's $t$ test. ACD=anterior chamber distance; TIA=trabecular-iris angle; AOD250 and AOD500=angle opening distance at $250 \mu \mathrm{m}$ and $500 \mu \mathrm{m}$ from the scleral spur; ILCD and ILCA=iris-lens contact distance and area. 
Table 2 Coefficients of variation (SD) (range) of ACD, TIA, AOD250, AOD500, and iris thickness wide angle and narrow angle groups

\begin{tabular}{lll}
\hline & Wide angle group & Narrow angle group \\
\hline ACD & $1.28(0.78)(0.33$ to 2.13$)$ & $2.13(1.25)(0.33$ to 3.73$)$ \\
TIA & $6.75(3.51)(1.38$ to 12.57$)$ & $9.05(4.25)(4.84$ to 17.03$)$ \\
AOD250 & $7.14(3.31)(3.28$ to 12.11$)$ & $9.78(4.96)(5.24$ to 18.33$)$ \\
AOD500 & $5.83(2.33)(1.87$ to 9.45$)$ & $8.78(3.55)(3.73$ to 14.67$)$ \\
Iris thickness & $6.31(3.16)(3.01$ to 10.11$)$ & $5.48(3.31)(2.82$ to 9.77$)$
\end{tabular}

$\mathrm{ACD}=$ anterior chamber distance; TIA=trabecular-iris angle; AOD250 and AOD500=angle opening distance at $250 \mu \mathrm{m}$ and $500 \mu \mathrm{m}$ from the scleral spur.
C

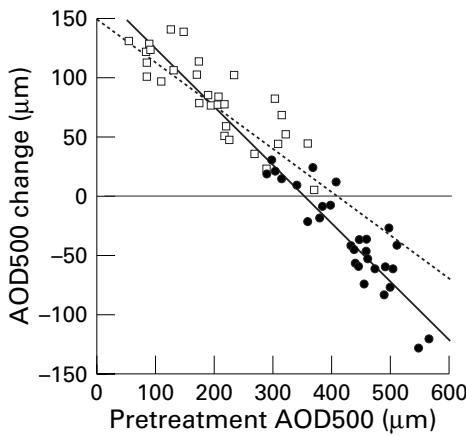

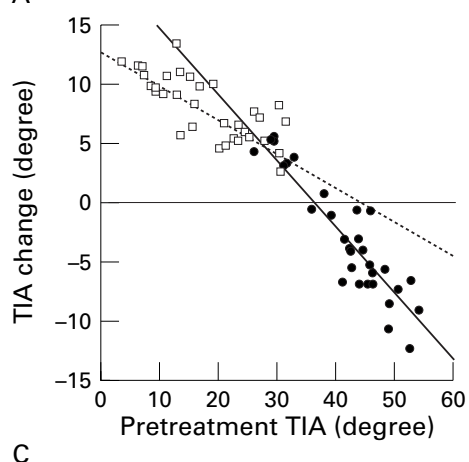

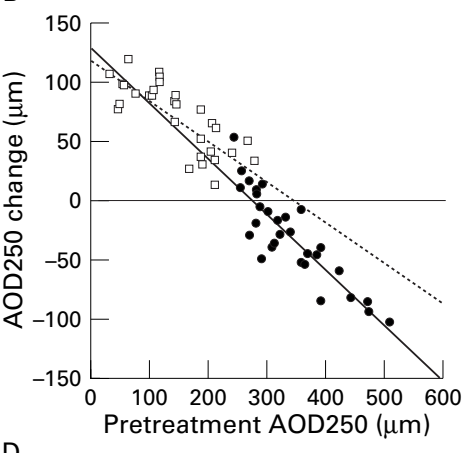

D

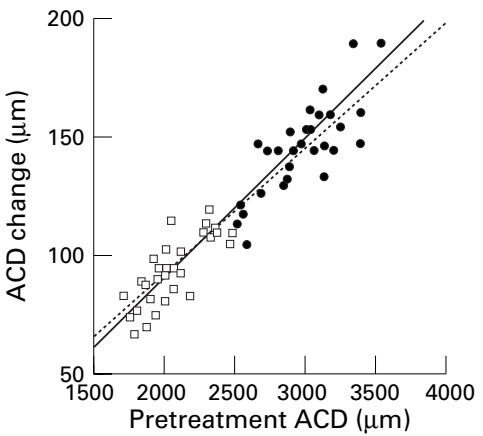

Figure 2 Relations between changes in TIA, AOD250, AOD500, and ACD and their own pretreatment values. (A) Change in trabecular-iris angle (TIA) plotted against pretreatment $T I A$. In narrow angle group, $r=0.807, p=0.0001$; change in $T I A=-0.289 \times$ pretreatment $T I A+12.789$; in wide angle group, $r=0.913, p=0.0001$; change in $T I A=-0.589 \times$ pretreatment TIA+21.220. (B) Change in AOD250 plotted against pretreatment AOD250. In narrow angle group, $r=0.787, p=0.0001$; change in $A O D 250=-0.333 \times$ pretreatment $A O D 250+119.360$; in wide angle group, $r=0.882, p=0.0001$; change in $A O D 250=-0.476$ $\times$ pretreatment $A O D 250+130.460$. (C) Change in AOD500 plotted against pretreatment AOD500. In narrow angle group, $r=0.852, p=0.0001$; change in $A O D 500=-0.352 \times$ pretreatment AOD500 + 151.861; in wide angle group, $r=0.895, p=0.0001$; change in $A O D 500=-0.494 \times$ pretreatment $A O D 500+176.174$. (D) Change in the anterior chamber depth (ACD) plotted against pretreatment $A C D$. In narrow angle group, $r=0.781, p=$ 0.0001 ; change in $A C D=+0.052 \times$ pretreatment $A C D-10.458$; in wide angle group, $r=$ $0.798, p=0.0001$; change in $A C D=+0.058 \times$ pretreatment $A C D-24.439$. Open symbols are narrow angle group; closed symbols are wide angle group.

laser iridotomy after this examination. There were 13 men and 17 women aged 63 to 82 years (mean 70.4 years). Exclusion criteria comprised the presence of high intraocular pressure, glaucomatous disc damage, plateau iris syndrome, anterior or posterior synechia, and ocular history, including glaucoma, trauma, inflammation, and previous ocular surgery. As controls, we studied 30 eyes of 30 age and sex matched healthy volunteers with

Table 3 Predictors of changes in angle width identified by multiple regression analysis

\begin{tabular}{lllll}
\hline Variable & $r$ & $r^{2}$ & F test & Linear regression equation \\
\hline ACD change & 0.769 & 0.592 & 21.737 & $+0.059 \times$ pretreatment ACD -28.725 \\
TIA change & 0.882 & 0.778 & 52.466 & $-0.031 \times$ pretreatment AOD $500+13.654$ \\
AOD250 change & 0.810 & 0.656 & 28.586 & $-0.403 \times$ pretreatment AOD250 +123.837 \\
AOD500 change & 0.908 & 0.824 & 70.116 & $-4.978 \times$ pretreatment TIA +159.603
\end{tabular}

$\mathrm{ACD}=$ anterior chamber distance; TIA=trabecular-iris angle; AOD250 and AOD500=angle opening distance at $250 \mu \mathrm{m}$ and $500 \mu \mathrm{m}$ from the scleral spur. wide angles (12 men and 18 women) ages between 65 and 85 years (mean 71.6 years). The study protocol and consent forms were approved by the human subjects committee of Amagasaki Hospital. Patients were informed of the purpose of our study, and provided their signed consent to participate. The diagnosis of narrow angle was based on the results of slit lamp biomicroscopy, gonioscopy, and ultrasound biomicroscopy. A narrow angle was diagnosed when trabecular-iris angle was $25^{\circ}$ or less on ultrasound biomicroscopic examination with grades III or IV angles based on Scheie's gonioscopic classification. ${ }^{24}$

We obtained ultrasound biomicroscopic measurements before and 1 hour after instillation of $2 \%$ pilocarpine hydrochloride (Santen, Osaka, Japan), as described by Kobayashi et al. ${ }^{8}$ The ultrasonic biomicroscope 840 (ZeissHumphrey, San Leandro, CA, USA) was used. Measurements were obtained three times to confirm reproducibility and to eliminate artefacts. Each subject was instructed to look at a mark placed on the ceiling to avoid the effects of accommodation. Room illumination was constant during the examination.

Anterior chamber depth (ACD) was measured as an axial distance from the internal corneal surface to the lens surface. The ultrasound biomicroscopic cross sectional views through the angle region were obtained for most of the measurements, which were performed in the temporal meridian; views were as vertical as possible, as determined from the screen image. The following variables were measured: ACD, trabecular-iris angle (TIA), angle opening distance at $250 \mu \mathrm{m}$ and $500 \mu \mathrm{m}$ from the scleral spur (AOD250 and AOD500), iris thickness, and iris-lens contact distance (ILCD) as described by Palvin et al. ${ }^{13}{ }^{14}$ Iris-lens contact area (ILCA) was measured as follows:

$\mathrm{ILCA}=\pi(\mathrm{R}+\mathrm{ILCD})^{2}-\pi \mathrm{R}^{2}$

where $\mathrm{R}$ is the distance between the centre of the anterior lens surface and the near point of iris-lens contact. Iris thickness was measured as the thickest part of the iris. Total axial length was measured by Ocuscan 4000 (Alcon, Fort Worth, TX, USA).

Values are expressed as mean (SD) and as a range. Unless otherwise specified, data were analysed by paired, unilateral $t$ tests. Multiple regression analysis was used to evaluate the contribution of individual factors to the change induced by pilocarpine in the ACD, TIA, AOD250, and AOD500. All the variables measured before and after the instillation of pilocarpine and the differences between them were considered as possible independent factors. To evaluate the reproducibility of the measurements, coefficient of variation was calculated. A level of $p<0.05$ was accepted as statistically significant. Data were stored on a personal computer. Statistical analysis was performed using the Statistical Analysing System (SAS). ${ }^{25}$

\section{Results}

Figure 1 shows a change of the angle by pilocarpine in an eye with a narrow angle. After 

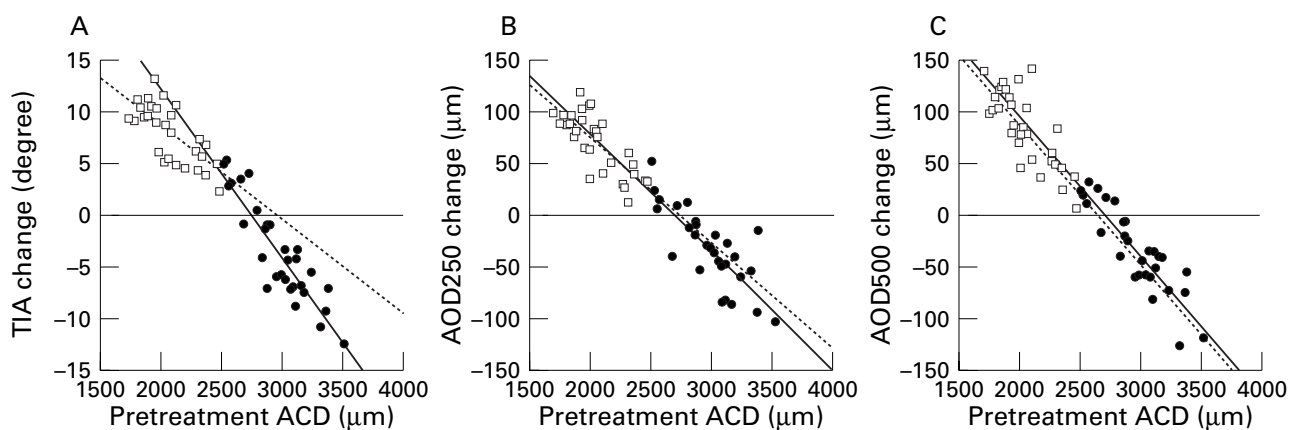

Figure 3 Relations between changes in TIA, AOD250, and AOD500 and pretreatment ACD. (A) Change in TIA plotted against pretreatment $A C D$. In narrow angle group, $r=0.706, p=0.0001$; change in $T I A=-0.009 \times$ pretreatment $A C D$ +26.726 ; in wide angle group, $r=0.907, p=0.0001$; change in TIA $=-0.016 \times$ pretreatment $A C D+45.043$. (B) Change in AOD250 plotted against pretreatment ACD. In narrow angle group, $r=0.769, p=0.0001$; change in $A O D 250=-0.105 \times$ pretreatment $A C D+286.073$; in wide angle group, $r=0.806, p=0.0001$; change in $A O D 250=$ $-0.115 \times$ pretreatment ACD +310.533 . (C) Change in AOD500 plotted against pretreatment ACD. In narrow angle group, $r=0.784, p=0.0001$; change in $A O D 500=-0.136 \times$ pretreatment $A C D+363.247$; in wide angle group, $r=$ $0.886, p=0.0001$; change in AOD500 = -0.135 $\times$ pretreatment $A C D+365.740$. Open symbols are narrow angle group; closed symbols are wide angle group.

the instillation of pilocarpine, ACD decreased from $2107 \mu \mathrm{m}$ to $2005 \mu \mathrm{m}$. TIA, AOD250, and AOD500 increased from $13.43^{\circ}$ to $29.22^{\circ}$, from $103 \mu \mathrm{m}$ to $192 \mu \mathrm{m}$, and from $120 \mu \mathrm{m}$ to $262 \mu \mathrm{m}$, respectively.

The means (SD) and ranges for age, sex, total axial length, ACD, TIA, and AOD250 and AOD500 before instillation of pilocarpine for eyes with narrow angle and those with wide angle are shown in Table 1 . Coefficients of variation of ACD, TIA, AOD250, AOD500, and iris thickness were shown in Table 2. No significant differences were found in age and sex between the two groups, but there were significant differences in total axial length, ACD, TIA, AOD250, and AOD500 ( $\mathrm{p}=$ $0.0017, \mathrm{p}=0.0001, \mathrm{p}=0.0001, \mathrm{p}=0.0001, \mathrm{p}$ $=0.0001$, respectively), as shown in Table 1 .

TRABECULAR-IRIS ANGLE (TIA)

All eyes in the narrow angle group showed a pilocarpine induced increase in TIA. Of 30 eyes with wide angles, 23 eyes, which had ACD $2670 \mu \mathrm{m}$ and more and TIA $35.6^{\circ}$ and more, showed a decrease in TIA. The mean change in TIA in the narrow group was significantly greater than that in the wide angle group $(\mathrm{p}=$ 0.0001 ) (Table 1). In both groups, the change in TIA following pilocarpine was inversely related to the pretreatment TIA, as shown in Figure 2A.

ANGLE OPENING DISTANCE AT 250 AND $500 \mu \mathrm{M}$ FROM THE SCLERAL SPUR (AOD250 AND AOD500) The AOD250 and AOD500 increased in all eyes with narrow angles. The AOD250 and AOD500 decreased in 23 of 30 eyes with wide angles. A statistically significant difference was found in the mean AOD250 and AOD500 between the narrow angle group and the wide angle group $(\mathrm{p}=0.0001 ; \mathrm{p}=0.0001)$ (Table 1). A significant relation between the pilocarpine induced AOD250 change and pretreatment AOD250 in the narrow angle and wide angle groups is illustrated in Figure 2B. Figure 2C illustrates a significant relation between the AOD500 change and pretreatment AOD500 in the narrow angle group and the wide angle group.

ANTERIOR CHAMBER DEPTH (ACD)

The mean decrease in ACD in the narrow angle group was 94 (SD 15) $\mu \mathrm{m}$, which was significantly less than that in the wide angle group $(\mathrm{p}=$ 0.0001) (Table 1). Percentage changes (for example, ACD change/pretreatment ACD)
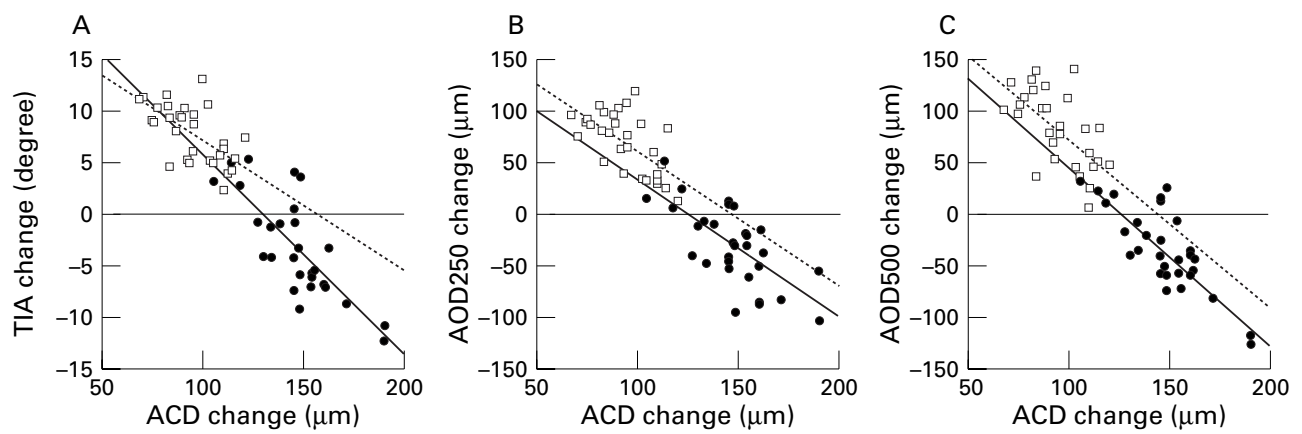

Figure 4 Relations between change in TIA, AOD250, and AOD500 and that in ACD. (A) Change in TIA plotted against change in $A C D$. In narrow angle group, $r=0.605, p=0.0004$; change in $T I A=-0.115 \times$ change in $A C D$ +18.778 ; in wide angle group, $r=0.791, p=0.0001$; change in $T I A=-0.197 \times$ change in $A C D+25.494$. (B) Change in AOD250 plotted against change in ACD. In narrow angle group, $r=0.632, p=0.0002$; change in AOD250 $=-1.264$ $\times$ change in $A C D+190.074$; in wide angle group, $r=0.694, p=0.0001$; change in AOD250 $=-1.368 \times$ change in $A C D+170.266$. (C) Change in AOD500 plotted against ACD change. In narrow angle group, $r=0.623, p=0.0002$; change in $A O D 500=-1.593 \times$ change in $A C D+232.895$; in wide angle group, $r=0.799, p=0.0001$; change in AOD500 $=-1.679 \times$ change in $A C D+212.380$. Open symbols are narrow angle group; closed symbols are wide angle group. 
were $4.95 \%(0.39 \%)(4.09-5.74 \%)$ in the narrow angle group and $4.57 \%(0.46 \%)(3.74-$ $5.64 \%)$ in the wide angle group, respectively. There was a significant difference between them. Significant linear correlations were found between pilocarpine induced change in ACD and the pretreatment ACD in the narrow angle group and the wide angle group, as shown in Figure 2D.

IRIS-LENS CONTACT DISTANCE AND AREA (ILCD, ILCA)

There was a significant difference in pretreatment ICLD and ICLA between eyes with wide angles and those with narrow angles ( $p=$ $0.0001, p=0.0001)$. A significant difference was found in pilocarpine induced ILCA change between the wide angle group and the narrow angle group ( $p=0.0006)$, whereas there was no difference in the ILCD change.

PREDICTION OF PILOCARPINE INDUCED CHANGE The most predictive variable of the change in ACD in the narrow angle group was pretreatment ACD, as shown in Table 3. The most predictive variable of the change in TIA was pretreatment AOD500 and that of the change in AOD250 was pretreatment AOD250. The most significant predictive variable of the change in AOD500 was pretreatment AOD500.

COMPARISON OF INDIVIDUAL FACTORS

A comparison of changes in individual variables with each other and pretreatment values revealed that the pretreatment ACD was correlated with the changes in TIA, AOD250, and AOD500, as shown in Figure 3. The changes in TIA, AOD250, and AOD500 showed statistically significant correlations to the change in ACD, as indicated in Figure 4.

\section{Discussion}

We evaluated the effects of pilocarpine on the angular width in 30 patients with narrow angles using ultrasound biomicroscopy; all eyes showed an increase in TIA, AOD250, and AOD500 in response to pilocarpine. These changes exhibited significant correlations with the corresponding pretreatment values. In control subjects whose anterior chamber depth was $2670 \mu \mathrm{m}$ and more and whose TIA was $35.6^{\circ}$ and more, pilocarpine produced decreases in TIA, AOD250, and AOD500. These changes increased linearly as the corresponding pretreatment values decreased. Where a CV $<10 \%$ was chosen as an acceptable reproducibility, the UBM system exhibited high reproducibility for all variables measured.

The pilocarpine induced responses of the ciliary muscle and outflow facility decline with age. ${ }^{26-28}$ Gabelt et al showed a decline of accommodation response to cholinergic drugs, decreased baseline outflow facility, and a decline in the facility response to pilocarpine hydrochloride in aging rhesus monkeys. ${ }^{26}$ Tamm et al reported an association between the age related decline in ciliary muscle mobility and the age related decline in outflow facil- ity and facility responsiveness to cholinergic drugs. ${ }^{28}$ We therefore selected age matched controls for our study.

The change in ACD induced by pilocarpine was significantly smaller in eyes with narrow angles than that observed in eyes with wide angles, and there was a significant difference in the percentage change between them. Resistance of the flow from the posterior chamber to the anterior chamber plays a major role in the response of the ACD, since the ciliary muscle motility and outflow facility responsiveness to pilocarpine decline in patients over $60 .{ }^{26-28}$ The resistance from the posterior chamber to the anterior chamber may relate to the iris-lens contact area or distance. An increase in the resistance induces an increase in the posterior chamber pressure and a decrease in the ACD. Pretreatment ILCD and ILCA of eyes with narrow angles were significantly greater than those of eyes with wide angles, indicating that eyes with narrow angles might show a greater resistance before pilocarpine instillation. Eyes with narrow angles showed a smaller pilocarpine induced increase in the ILCA than eyes with wide angles, whereas there was no difference in the ILCD. Eyes with narrow angles appeared to have a smaller increase in the resistance, and showed a smaller decrease in the ACD. The change in the resistance depends on the pretreatment ACD. The importance of such pretreatment values has not yet been addressed. Abramson et al showed that, in elderly subjects, pilocarpine induced a decrease of $50-200 \mu \mathrm{m}$ in the ACD. ${ }^{29}$ These variable decreases may have been attributable to differences in pretreatment ACD.

Changes in the anterior chamber angle opening are caused by two types of reactions - a forward movement of the iris plane and a pull on the iris root and a decrease of the total thickness of the iris. In this study, no difference was found in pilocarpine induced change of iris thickness between the narrow and wide angle groups. Pilocarpine may induce a smaller decrease in the ACD in eyes with narrow angles than in those with wide angles, leading to less forward movement of the iris plane in eyes with narrow angles. In eyes with narrow angles, an increase in the angle width caused by the thinning of the iris was greater than a decrease in the angle by forward movement of the iris plane.

Multiple regression analysis showed that even in patients over the age of 60 with narrow angles, the change in angle opening and ACD could be predicted before instillation of pilocarpine. This predictive factor may be useful to ophthalmologists in treating those patients. Because pilocarpine induced widening of the angle in patients with narrow angles, it is a good candidate for temporal treatment of patients with narrow angles and angle closure glaucoma. The use of ultrasound biomicroscopy facilitates the evaluation of the angle before and after instillation of pilocarpine. However, long term response to pilocarpine remains unclear. The mechanism of pilocarpine induced change in the angle requires further investigation. The pilocarpine induced 
changes with age also need further study because of the change of outflow facility and accommodation with age.

In summary, we demonstrated the effects of pilocarpine on the anterior chamber angle in patients with narrow angles. We showed that in all eyes with narrow angles pilocarpine induced widening of the angle. We also demonstrated a significant correlation between pilocarpine induced change in the angular width and pretreatment state.

1 Abramson DH, Coleman DJ, Forbes $\mathrm{M}$ et al. Pilocarpine, effects on the anterior chamber and lens thickness, Arch Ophthalmol 1972;87:615-20.

2 Mindel JS, Kharlamb AB. Alteration of acetylcholine synthesis by pilocarpine. Arch Ophthalmol 1988;102:154650 .

3 Armaly MF, Jepson NC. Accommodation and the dynamics of the steady-state intraocular pressure. Invest Ophthalmo 1952;1:480-5.

4 Van Buskirk EM. The canine eye: lens depression and aqueous outflow. Invest Ophthalmol Vis Sci 1980;19:789-93.

5 Moses RA, Grodzki WJ Jr. Choroid tension and facility of aqueous outflow. Invest Ophthalmol Vis Sci 1977;16:10625 .

6 Kaufman PL, Barany EH. Residual pilocarpine effects on outflow facility following surgical disinsertion and retrodisplacement of the ciliary muscle from the scleral spur in the placement of the ciliary muscle from the scieral spur in the

7 cynomolgus monkey. Invest Ophthalmol 1976;15:558-62. 7 .

8 Kobayashi H, Kobayashi K, Kiryu J, et al. Ultrasound biomicroscopic analysis of the effect of pilocarpine on the anterior chamber angle. Graefes Arch Clin Exp Ophthalmol 1997;235:425-30.

9 Makabe R. Vergleichende Untersuchungen der Kammerwinkelweite mit Echographie und Gonioscopie. Klin Monatsbl Augenheilkd 1989;194:6-9.

10 Jin JC, Anderson DR. The effect of iridotomy on the iris contour. Am f Ophthalmol 1991;110:260-3.

11 Tiedeman JS A physical analysis of the factors that determine the contour of the iris. Am $f$ Ophthalmol 1991;111:338-43.

12 Anderson DR, Jin JC, Wright MM. The physiologic characteristics of relative pupillary block. Am $\mathcal{F}$ Ophthalmol 1991; 111:344-50.

13 Pavlin CJ, Harasiewicz K, Foster FS. Subsurface ultrasound microscopic imaging of the intact eye. Ophthalmology 1990; microscopic in $244-50$.
14 Pavlin CJ, Harasiewicz K, Sherar MD, et al. Clinical use of ultrasound biomicroscopy. Ophthalmology 1991;98:28795.

15 Pavlin CJ, Harasiewicz K, Foster FS. Ultrasound biomicroscopy of anterior segment structures in normal and glaucomatous eyes. Am f Ophthalmol 1992;113:381-9.

16 Pavlin CJ, McWhae JA, McGowan HD, et al. Ultrasound biomicroscopy of anterior segment tumors. Ophthalmology 1992:99:1220-8.

17 Pavlin CJ, Ritch R, Foster FS. Ultrasound biomicroscopy in plateau iris syndrome. Am f Ophthalmol 1992;113:390-5.

18 Tello C, Chi T, Liebmann J, et al. Ultrasound biomicroscopy in pseudophakic glaucoma. Ophthalmology 1993;100:13304.

19 Potash CJ, Tello C, Liebmann J, et al. Ultrasound biomicroscopy in the pigment dispersion syndrome. Ophthalmology 1994;101:332-9.

20 Tello C, Liebmann J, Potash SD, et al. Measurement of ultrasound biomicroscopy images: Intraobserver and interobserver reliability. Invest Ophthalmol Vis Sci 1994;35: 3549-52.

21 Ailanides IM, Libre PE, Silverman RH, et al. High frequency ultrasound imaging in pupillary block glaucoma. Br f Ophthalmol 1995;79:972-6.

22 Wilensky JT. Should patients with anatomically narrow angles have prophylactic iridectomy? 1. Narrow angles accompanied by slit-lamp and gonioscopic evidence of risk are indications for prophylactic laser iridectomy. Surv Ophthalmol 1996;41:31-2.

23 Wilensky JT. Should patients with anatomically narrow angles have prophylactic iridectomy? 2. Definitive signs and gonioscopic visualization of appositional angle closure are indications for prophylactic laser iridectomy. Surv Ophthalmol 1996;41:31-2.

24 Scheie HG. Width and pigmentation of the angle of the anterior chamber. A system of grading by gonioscopy. Arch Ophthalmol 1957;58:510-5.

25 SAS Institute Inc. SAS/STAT guide for personal computers, Version 6. Cary, NC: SAS Institute Inc, 1989.

26 Gabelt BT, Crawford K, Kaufman PL. Outflow facility and its response to pilocarpine decline in aging rhesus monkeys. Arch Ophthalmol 1991;109:879-82.

27 Lutjen Drecoll E, Tamm E, Kaufman PL. Age-related loss of morphologic responses to pilocarpine in rhesus monkey ciliary muscle. Arch Ophthalmol 1988;106:1591-8.

28 Tamm E, Croft MA, Jungkunz W, et al. Age-related loss of ciliary muscle mobility in the rhesus monkey. Role of the choroid. Arch Ophthalmol 1992;110:871-6.

29 Abramson DH, Franzen LA, Coleman DJ. Pilocarpine in the presbyope. Demonstration of an effect on the anterior chamber and lens thickness. Arch Ophthalmol 1979;89: $100-2$. 